\title{
Perspectives on External Support to Low Level Private Health Facilities in Management of Childhood Infections in Mbarara District, Uganda: A Qualitative Study With Health Workers and Policy Makers
}

Juliet Mwanga-Amumpaire ( $\nabla$ jmwanga@must.ac.ug)

Mbarara University of Science and Technology https://orcid.org/0000-0002-6738-477X

Joan Nakayaga Kalyango

Makerere University College of Health Sciences

Karin Källander

Karolinska Institutet

Radhika Sundararajan

Department of Emergency Medicine, Weill Cornell Medicine, New York

Judith Owokuhaisa

Mbarara University of Science and Technology

Celestino Obua

Mbarara University of Science and Technology

Tobias Alfvén

Karolinska Institutet

Grace Ndeezi

Makerere University College of Health Sciences

Research article

Keywords: Low-level, private, health care, support, childhood infections, policy makers, qualitative, interviews, paediatric care.

Posted Date: September 8th, 2020

DOI: https://doi.org/10.21203/rs.3.rs-63967/v1

License: (c) (i) This work is licensed under a Creative Commons Attribution 4.0 International License.

Read Full License 


\section{Abstract}

Background: With the under-five child mortality rate of 46.4 deaths per 1000 live births, Uganda needs to accelerate measures to reduce child deaths in order to achieve the Sustainable Development Goal 3. While $60-70 \%$ of frontline health services are provided by the private sector, many low level private health facilities are unregistered, unregulated, and often miss out on innovative strategies rolled out by the Ministry of Health. Low level private health facilities need support in order to provide quality health care. We explored the perspectives of health workers and policy makers on external support given to low level private clinics providing health care for children.

Methods: In-depth interviews were conducted from May to December 2019 with 43 purposively selected key informants. They included 30 health care professionals treating children in low level private clinics and 13 policy makers from Mbarara district and the Uganda Ministry of Health directly involved with ensuring quality of child health. The issues discussed included their views on the quantity, quality, factors determining support received and preferred modalities of support to low level private health facilities. Using an inductive approach, interview transcripts were coded to identify categories and themes.

Results: We identified three themes which emerged from the data 1) External support is needed to address socio-economic, regulatory and knowledge gap issues, 2) Current support is not optimal, and, 3) Ideal support underscores working together. While the Ministry of Health recognises its' responsibility to provide support and guidance to public and private health facilities, it acknowledges lack of support for low level private health facilities currently. Health providers emphasised technical capacity building and more supportive supervisory visits but not simply policing and apportioning blame.

Conclusion: The current support being given to low level private health facilities (LLPHF) is inadequate. The support needs to be tailored to the needs of the facility and health facilities have to proactively ask for support. Capacity building with emphasis on training and supportive supervision are key strategies for providing external support to LLPHF.

\section{Background}

In low income countries of Sub-Saharan Africa, one child out of twelve dies before their 5th birthday, compared to one out of 147 in high income countries [1,2]. With the under-five child mortality rate of 46.4 deaths per 1000 live births [3] Uganda is one of the countries where acceleration of progress is needed in order to achieve the Sustainable Development Goal (SDG) 3 of reducing under-five mortality to 25/1000 live births by 2030 . Over $70 \%$ of the deaths among the under-fives are due to treatable infectious causes namely malaria, pneumonia, neonatal infections and diarrhoeal diseases [4].

The Ministry of Health $(\mathrm{MOH})$ of Uganda recommended implementation of the Child Survival strategies in a bid to achieve the SDGs. These strategies include new-born care, infant and young child feeding, use of long-lasting insecticide treated mosquito nets to prevent malaria, immunization including introduction of newer vaccines such as the pneumococcal conjugate vaccine and management of common childhood 
illnesses at all levels of health facilities [4]. However poor uptake and implementation of these interventions at health facilities due to inadequate supervision and technical support, and lack of resources to effectively assimilate the strategies in district activities, are important barriers to achieving reductions in childhood mortality [5].

While public health facilities provide free services, are regulated and should be fully supported by the $\mathrm{MOH}$, not everybody seeks health care from them. Prior research has described several factors which influence utilization of private facilities, including inadequate medicines and regular stock outs at public facilities, long waiting times, unfriendly healthcare providers, poor sanitation, non-availability of healthcare providers over weekends and at night, facilities opening only on some selected days, health worker demand for money for treatment, and location of health facilities away from villages where people reside $[4,6,7]$.

Therefore, private health facilities play an important role in providing health care to children in Uganda. However, some private facilities function independent from the district health team, as they are often unregistered. Like in other lower income countries, private health facilities are socially stratified and low level private facilities may be unregulated $[8,9]$. The $\mathrm{MOH}$ recognizes the difficulty to quantify the input of private sector to provision of health for Ugandans because of poor reporting and non-inclusion of data from private sector health facilities in the National Health Management Information System [10-12]. However, the Uganda Demographic and Health Surveys indicate that $60-70 \%$ of frontline health services and $50-60 \%$ of care for febrile children are provided by private primary level health facilities [4]. Strategies for management of childhood illness rolled out by the $\mathrm{MOH}$ have targeted mainly public institutions, often leaving out the private providers.

Private health facilities have been described as often violating medical standards of practice, sometimes resulting in poorer patient outcomes[13]. Some of them exaggerate incentives from unnecessary testing and treatment, and may have poor infrastructure with inadequate clinic space, lack basic diagnostic instruments such as thermometers, and employ health workers with poor clinical competence $[11,13]$. Sub-optimal quality of care from these facilities may contribute to sustained mortality and morbidity from easily treatable diseases. While a survey carried out in Eastern Europe, Asia, Latin America and Africa showed that provision of practice-support tool kits, such as guidelines, protocols and standard operating procedures can improve quality of care, other studies have demonstrated that mere availability of treatment guidelines in a facility will not automatically improve care for sick children [14-16]. In order to reduce childhood mortality in Uganda, there is an urgent need to understand how private health facilities can be supported in managing paediatric illness so that the treatment given to children is safe and evidence based. The goal of this study was to explore the perspectives of health care providers in low level private health facilities (LLPHF) and policy makers, concerning the external support provided to LLPHF in the management of common childhood infections.

\section{Methods}




\section{Research design and setting}

This qualitative study was carried out among health care providers in private clinics managing sick children in Mbarara District and policy makers in Mbarara District and the $\mathrm{MoH}$ in Uganda. Mbarara is located $267 \mathrm{~km}$ south-west of Kampala, Uganda and includes 3 administrative counties, 16 sub-counties, 83 parishes and 742 villages. The district is primarily rural with a population of about 470,000 inhabitants and a density of 99 inhabitants $/ \mathrm{km}$. The main health facility is Mbarara Regional Referral Hospital, a tertiary referral centre that also serves as a teaching hospital for the medical school of Mbarara University of Science and Technology. The health facilities in the district follow the national ministry of health facility classification defined by the catchment area served and the services offered as illustrated in table 1 below [17].

Table 1. The health facility classification in Uganda

\begin{tabular}{|lll|}
\hline $\begin{array}{l}\text { Level of } \\
\text { Health } \\
\text { Unit }\end{array}$ & $\begin{array}{l}\text { Target } \\
\text { population }\end{array}$ & Services provided and structures \\
$\begin{array}{l}\text { Village } \\
\text { Health } \\
\text { Teams } \\
\text { (Health } \\
\text { Centre I) }\end{array}$ & $1,000$. & $\begin{array}{l}1^{\text {st }} \text { contact for populations living in rural areas providing community-based } \\
\text { preventive and health promotion services, community mobilization and } \\
\text { referral of sick members to health facilities. No physical structures }\end{array}$ \\
$\begin{array}{l}\text { Health } \\
\text { Centre II }\end{array}$ & 5,000 & $\begin{array}{l}\text { Parish level facility offering Disease Prevention, Health Promotion and } \\
\text { Outpatient Curative Health Services for uncomplicated conditions, } \\
\text { antenatal care and immunisation for children. }\end{array}$ \\
$\begin{array}{l}\text { Health } \\
\text { Centre III }\end{array}$ & 20,000 & $\begin{array}{l}\text { Sub county level facility offering Preventive, Health Promotion, Outpatient } \\
\text { Curative, Maternity, inpatient Health Services and Laboratory services for } \\
\text { malaria testing and tuberculosis microscopy }\end{array}$ \\
$\begin{array}{l}\text { Health } \\
\text { Centre } \\
\text { IV }\end{array}$ & 100,000 & $\begin{array}{l}\text { County level facility offering disease Preventive services, Health Promotion, } \\
\text { Outpatient Curative, Maternity, inpatient Health Services, Emergency } \\
\text { surgery and Blood transfusion and Laboratory services }\end{array}$ \\
$\begin{array}{l}\text { General } \\
\text { Hospital }\end{array}$ & 500,000 & $\begin{array}{l}\text { District level facility, In addition to services offered at HC IV, offers general } \\
\text { services and in-service training, consultation and research to community } \\
\text { based health care programs. }\end{array}$ \\
\hline $\begin{array}{l}\text { Regional } \\
\text { Referral } \\
\text { Hospital }\end{array}$ & $2,000,000$ & $\begin{array}{l}\text { In addition to services offered at the general hospital, offers specialist } \\
\text { services, such as psychiatry, Ear, Nose and Throat, Ophthalmology, } \\
\text { dentistry, intensive care, radiology, pathology, higher level surgical and } \\
\text { medical services. }\end{array}$ \\
\hline $\begin{array}{l}\text { National } \\
\text { Referral } \\
\text { Hospital }\end{array}$ & $10,000,000$ & $\begin{array}{l}\text { Offers comprehensive specialist services and are involved in teaching and } \\
\text { research. }\end{array}$ \\
\hline
\end{tabular}

There are 7 hospitals in the district, of which 6 are private. Most of the health centres $(\mathrm{HC})$ are public and 
these include $4 \mathrm{HC} \mathrm{IV}, 16 \mathrm{HC}$ III and $29 \mathrm{HC}$ II facilities. One hundred and twenty-four private facilities were registered with the national regulatory authorities in 2017 and are homogenously distributed within the urban and rural settings. The majority of private health facilities in Mbarara are at $\mathrm{HCIII} \mathrm{level} \mathrm{and} \mathrm{lower.} \mathrm{In}$ the context of this study we defined such facilities as low level private health facilities (LLPHF). They have minimal infrastructure and treat common diseases of children such as uncomplicated malaria, pneumonia and diarrhoea and may provide immunization services for children. A few have small inpatient services limited to a maternity ward and a laboratory for simple diagnostics like malaria blood slides and urine tests. Many private clinics are run by nurses, midwives and clinical officers whose highest level of qualification are certificates or diplomas, while a few are headed by medical officers with medical degrees. In more rural facilities, the services may even be delivered by individuals with minimal or no prior training in health-related fields.

\section{Sampling and recruitment}

Purposive sampling of health care providers and policy makers was used to identify key informants with rich experiences pertinent to the study topic. To capture the heterogeneity among the 30 health care providers who participated in the interviews, 10 were included per county; 1 per health facility. They ranged from nursing assistants, nurses/midwives, clinical officers and medical doctors. Inclusion criteria for the providers were: 1) providing clinical care for children in private settings for at least 6 months; and 2) being employed at a LLPHF. We also recruited 13 key policy makers using the following inclusion criteria: 1) employed at their post for at least 2 years; and 2) directly involved with ensuring quality of child health in the district or $\mathrm{MoH}$ headquarters. The policy makers included a broad range; one participant from the Quality Assurance Department of the $\mathrm{MoH}$, five from Mbarara District Health Office (DHO) and seven from the health sub-districts (HSD). HSDs are the lowest Ministry of Health administrative structure and directly supervise the lower public health facilities and are hierarchically supervised by the DHO. These key informants were identified as such by the cooperation of their supervisors. All individuals who were approached accepted to participate in the study. The sample size of 43 participants was determined through data saturation, or the point when additional interviews did not provide novel insight or point to new concepts $[18,19]$. All participants provided written informed consent, including permission to audio-recorded interviews. After completion of the interview, participants received 20,000 Uganda Shillings ( $\$ 5$ United States dollars) as compensation for participation, the minimum standard for research studies in the country.

\section{Data collection}

This research was part of a bigger study investigating the quality of care for childhood infection in Mbarara district in which data was collected from May to December 2019. The in-depth interviews were conducted by two female (BK and PT) and 1 male (CO) Ugandan research assistants (RAs) with prior training and experience in carrying out qualitative interviews and fluent in English and Runyankore, the dialect spoken by majority of people in Mbarara. Prior to the study initiation, the RAs were trained for 3 days on the study protocol, principles of qualitative data collection and how to conduct high-quality 
interviews, interview translation and transcription. An interview guide was specifically created for this study to ensure consistent focus on the following topics across all interviews: 1) views on the quantity and quality of support received by LLPHF 2) factors determining support received by the LLPHF; and 3) preferred modalities of support. RAs were trained intensively on the interview guide and participated in validating this guide by piloting it with 3 participants whose responses were not included in the analysis. The interviews were conducted at the respondents' respective work places, in a quiet and private room, at a time and in a language that was convenient to them. Each interview lasted approximately 60 minutes. Most of the interviews were carried out in the English language per participant request. All interviews were transcribed verbatim, based on the audio recordings. For those interviews conducted in Runyankore, the transcription and translation into English was carried out by the RAs. All transcripts were created in English for analysis and proof read by author JMA who speaks both languages fluently.

The interview quality was monitored by JMA who received and read the transcripts line by line within 48 hours of transcript completion, and provided feedback to the 3 RAs to continuously improve their interview skills throughout the data collection period. This ensured consistency in quality and content across all interviews, and served as a means to monitor for data saturation.

\section{Analytical process}

Interview data was analysed using content analysis [20]. The interviews were considered iteratively in order to facilitate exploration of emerging themes and to actively monitor for data saturation. Author JMA reviewed transcripts within 48 hours of completion to identify the emerging concepts. Following an 'open coding' process [21] preliminary set of codes were developed. The codes assigned were grounded in the raw data, and merged into relevant categories and themes to address the research objectives. JMA reviewed and refined the codes constantly as new transcripts were received using a constant comparison analysis method. Another co-author ( $\mathrm{JO}$ ) reread the transcripts and independently created a set of codes. After comparison of the codes the 2 authors came up with the final set through discussion and consensus.

ATLAS.ti (GmbH, Berlin) was used for data organisation. Quotes from the participants are presented to illustrate corresponding themes and are italicised and indented.

\section{Results}

The characteristics of the 43 participants are shown in Table 2 below. Majority had been in service for more than 1 year. 
Table 2

Characteristics of the study participants

\begin{tabular}{|c|c|c|}
\hline Characteristic & $\begin{array}{l}\text { Private health care workers } \\
\mathrm{N}=30, \mathrm{n}(\%)\end{array}$ & $\begin{array}{l}\text { Policy makers } \\
N=13, n(\%)\end{array}$ \\
\hline \multicolumn{3}{|l|}{ Gender } \\
\hline Female & $12(40)$ & $6(46)$ \\
\hline Male & $18(60)$ & $7(54)$ \\
\hline \multicolumn{3}{|l|}{ Duration in service } \\
\hline 6 months to 1 year & $4(13)$ & $0(0)$ \\
\hline$>1$ to 5 years & $12(40)$ & $3(23)$ \\
\hline$>5$ years & $14(47)$ & $10(77)$ \\
\hline \multicolumn{3}{|l|}{ Profession } \\
\hline Nursing assistant & $3(10)$ & $0(0)$ \\
\hline Nurse/Midwife & $17(57)$ & $5(39)$ \\
\hline Clinical Officer & $6(20)$ & $3(23)$ \\
\hline Medical doctor & $3(10)$ & $2(15)$ \\
\hline Other* & $1(3)$ & $3(23)$ \\
\hline
\end{tabular}

Our inductive data analysis results are summarised in Table 3 and indicated there was some level of external support being given to LLPHF albeit being far from ideal in extent and quality. It indicates barriers to provision of support as well as what would be the ideal way for support in this context. We describe three over-arching themes which emerged from the data: 1) external support is needed, 2) current support is not optimal, and, 3) ideal support underscores all stakeholders working together. 
Table 3

Summary of the emerging themes and categories

\begin{tabular}{|c|c|c|c|}
\hline Themes & $\begin{array}{l}\text { External support is } \\
\text { needed }\end{array}$ & $\begin{array}{l}\text { Current support is } \\
\text { not optimal }\end{array}$ & $\begin{array}{l}\text { Ideal support underscores } \\
\text { working together }\end{array}$ \\
\hline $\begin{array}{l}\text { Categories and } \\
\text { sub-categories }\end{array}$ & $\begin{array}{l}\text { - Solving socio- } \\
\text { economic issues } \\
\text { - Bridging providers' } \\
\text { knowledge gaps } \\
\text { - Address regulatory } \\
\text { issues. }\end{array}$ & $\begin{array}{l}\text { - Limited support } \\
\text { - Punitive rather } \\
\text { than supportive } \\
\text { - Barriers to support } \\
\text { o Ignorance } \\
\text { o Negative attitudes } \\
\text { o Unfounded fear } \\
\text { o Bureaucracies } \\
\text { o Inaccessibility of } \\
\text { facilities } \\
\text { o Limited resources }\end{array}$ & $\begin{array}{l}\text { - Capacity building by } \mathrm{MoH} \\
\text { and partners } \\
\text { o training } \\
\text { o technical support } \\
\text { o support supervision } \\
\text { - Provision of materials and } \\
\text { drugs }\end{array}$ \\
\hline
\end{tabular}

\section{External support is needed}

External support was described as necessary for LLPHF to address: 1) patients' socio-economic issues 2) providers' knowledge gaps, and, 3) regulatory issues.

Operational costs for running private clinics are transferred to patients as clinics do not receive subsidies. For some patients these medical costs are prohibitively expensive therefore caretakers self-medicate their unwell children, leading to misuse of drugs. Subsidising costs will encourage parents to bring their children for medical care in time;

".... when someone comes and the bill is beyond one hundred thousand Shillings (खUSD 26) and yet has less than that... the next day he will not come back when he falls sick. That is why people have resorted to self-medication. When a child has a fever, they 'pump' Panadol (paracetamol)..... by the time they get to hospital its already a complicated case but if that immediate care was there at a cheaper cost or even at a free cost in some private centre...it will help a lot." (Male medical doctor, urban clinic)

While many health workers are knowledgeable and doing their best in the context they work, respondents pointed out that there are some who have inadequate knowledge to treat childhood conditions because of lack of opportunities to update their knowledge or inadequate qualifications. Some carry out unethical practices knowingly or unknowingly and some health facilities are not registered and are carrying out the activities illegally. These points are illustrated in these quotes;

"Yes, we have realized that most of the people managing these facilities are staff of low education level like enrolled nurses, lab assistants and a few clinicians... we need to give them basic ideas, trainings on 
management of childhood illness... we need to find ways of strengthening the supportive supervision..." Official from $\mathrm{DHO}$

"...most health workers working in private sector rarely receive trainings so which means there is a knowledge gap....the care they offer may not be in line with the standards of the $\mathrm{MOH}$. They are not regularly supervised they may think that whatever they do is right..."Official from HSD

"Yes, sometimes you find quack (health) workers dressed in white gowns being called doctor yet has never trained as a health worker.... Whistle blowers write letters (to the DHO)...that someone has died from this clinic. When you investigate, you find that the clinic is not in the district registry...." (Official from DHO)

\section{Current support is sub-optimal}

It was acknowledged that some external support is already being extended to LLPHF, however it is not yet ideal, both in extent and quality. Support is provided by the district health office, $\mathrm{MoH}$ affiliated bodies such as National Drug Authority (NDA) and some non-government organisations (NGOs). Two categories describe the current support: 1) The support is limited, and 2) supervision is punitive rather than supportive.

The DHO facilitates immunisation activities and, issues registers and clinical guideline booklets, but the support is inconsistent and not all clinics benefit. Other entities like private pharmacies and nongovernment organisations give limited in-kind support, to promote organisational agendas. The Ministry of Health recognises that it is its responsibility to provide support and guidance to public and private health facilities, but acknowledges lack of support especially for LLPHF.

"The national level does a lot of development of policies, standards and guidelines. The weakness is we do not disseminate them well and do not ensure that they are used well.... we have very good guiding documents but the actual dissemination and their use at lower level is not well monitored..." (MoH official)

"Support supervision is done in government health facilities however some registered private facilities are supported by some NGOs like Blue Star; those clinics that do deliveries are given vaccines and mama kits but for us we are not considered." (Female nurse, rural clinic)

Capacity building is rarely extended to practitioners in LLPHF; opportunities are offered mainly to health workers in public facilities or big Private-Not-for-Profit hospitals. The LLPHF rarely get to know about new guidelines and policies. Due to limited funding at the DHO only select facilities registered with the DHO or supported by private implementing partners receive supervisory visits. The ministry officials or district health officials mainly visit clinics to check for licensure and stolen government drugs, but not to offer technical support.

"At the moment we go there when there is a problem; even the implementing partners are not able to support the private facilities because it's not in their mandate, so even if there is a problem it's more 
comfortable to first go to the government facility, then pass by the private one as a by-the-way. Since it is a by-the-way service you don't expect much results. The time and effort put in are limited." (DHO official)

"They have never come to support, they only come to check whether you have fulfilled the requirements like registering or if you renewed the practicing license, but concerning support not at all." (Male nurse, rural clinic)

"Currently they are so much interested to know whether we have the government things around...they don't want to see if we are segregating the waste well they only want to see if we have Panadol that has government of Uganda mark that is what is in their heads..." (Male clinical officer, urban clinic)

Ignorance, negative attitudes and fear by private health providers, bureaucracies, inaccessibility of facilities and limited resources at the district, are hindrances to provision of optimal support. Support supervisory visits are misconceived by some private health workers who fear the consequences of lacking appropriate documents to run a private clinic or monetary compensation for the technical support provided. Health workers exhibit negative attitudes to use of national guidelines and make no effort to access such support even where it has been offered by the DHO. In addition LLPHF find the criteria to qualify for $\mathrm{MoH}$ support such as registration and association with the Uganda Health Federations complex.

"...small clinics are not aware that they need to be affiliated to bureaus, they are not aware of the health federation for the private sector. They don't even know that they can get funding and be supported or even be trained for this.... all that information is not passed to them. And then...since they are businesses some of them do not mind, and because some are not well trained; they don't want to be known". (MOH official)

"If they give us support supervision) won't they charge us money? Won't we get problems?" (Female nursing assistant, rural clinic)

Poor terrain and failure of facility staff to avail themselves is a further cumbrance to support supervision. The district does not plan for them because it does not know that they exist;

"...in my place, it is very hilly and difficult to access those small health facilities, district people don't go there. They stop at the facilities which are easily accessible" (Nursing officer, HSD)

"Majority of the providers don't have papers.... When they are supposed to be supervised, they hide. They always fear a lot.... they are suspicious even when there is no harm....." (Male doctor urban clinic)

The policy makers named limited human resources and funding as constraints to supporting private clinics;

"....there used to be vehicles... for support supervision but these days they are rare, you go at the whole district and find only one motorcycle..." (DHO official) 
While all respondents said that it is the mandate of the $\mathrm{MoH}$ to provide support to health facilities they noted that everybody including facility owners and other partners in health have a responsibility. Figure 1 illustrates how the preferred support for the LLPHF can be organised showing the different roles of all stakeholders.

Preferred support includes 1) capacity building by training and technical supportive supervision; 2) provision of materials and drugs. The health workers and policy makers emphasised refresher training as an important support and mandatory training on evidence-based strategies, and professionalism that should be provided by the $\mathrm{MoH}$ or other partners for continuous skills improvement. Many respondents felt that the supervisory visits should be more supportive than simply policing and apportioning blame. They prefer that $\mathrm{MoH}$ oversees the supervision of private health facilities, sets the standard of care, gives guidelines, enforces regulation and streamlines payment and salaries across the private facilities. They want the DHO and professional councils to offer support supervision while NGOs offer other nonsupervisory support.

"...incorporate this IMCl guidelines into the training of most of the health professionals because some children are seen by the lower carders. The IMCl guidelines are not hard, if we can roll them out in as many facilities and even lower cadres the better.... that would improve the care.... and still of course training people not to look at the money only but look at the holistic care of the children...." (Male doctor urban clinic)

"Let it be regular and sustainable, unless we befriend them we may not get information from them.... because for NDA they go there but are very rude..... we need to be friendly if we are to improve them." (Clinical officer, HSD)

"....if government decides.... this is the level of management of our children that we need you make sure that is effected, put up management standards in these facilities and give the guidelines. But you find some people cannot access the guidelines... they are working 10 years and never been supervised. (Male doctor, urban clinic)

Sensitisation of caretakers by $\mathrm{MoH}$ to trust private health facilities so that they seek care in time in addition to support with materials, medicines and subsidising operation costs of private clinics are some of the ways facilities want to be supported;

"...the district and the entire government should do that task of sensitising the public... to trust clinics because for us in the private sector once we try to tell the public they will think that we are just trying to get the opportunity of getting money...." (Male nurse urban clinic)

"The government should supply quality drugs for children at a low price this will help parents who are not able to pay high bills. Clinics are nearer to people but some fear to take their children there for treatment because of charges and end up losing their children. (Female nurse, rural clinic) 


\section{Discussion}

In this study we identified reasons for need of external support by LLPHF which included overcoming socio-economic as well as regulatory challenges and improving health provider knowledge. The current support is considered sub-optimal, however, it is a good starting point from which the leadership of the $\mathrm{DHO}$ and $\mathrm{MoH}$ can build upon and ensure the support reaches the facilities at the lower level of service provision. Support that is provided can only be effective if the end users take it up. Barriers to uptake and provision of support include wrong provider attitudes and ignorance of the health workers towards support supervision, limited resources, and inaccessibility of facilities.

Often, the poor people in the rural areas may not afford fees charged for services provided by private health providers and so subsidising these costs by the government and other entities could improve health care seeking behaviours of the population as delays in seeking care has been described by other researchers as one of the factors contributing to child deaths [22]. Subsidising medical costs is possible as it has already been piloted in selected districts in northern, eastern and western Uganda to increase access to quality obstetrics and new-born services [23]. In Japan, introduction of a medical subsidy scheme for school children increased utilisation by children without chronic illnesses [24]. In low resource settings, the more qualified providers mainly work in urban facilities while some rural health facilities, have underqualified cadres $[9,11]$. Our study found that LLPHF in Uganda are often left out of trainings and technical support. Including LLPHF in capacity building programs of the $\mathrm{MOH}$ is important in overcoming the knowledge gap challenge.

Current external support extended to LLPHF is considered non-optimal. LLPHF are left out in many aspects and communications on how to access support from the MOH rarely gets down to LLPHF who also find the conditions that have to be fulfilled before accessing support complex. Other studies have also described complex rules and inadequate communication concerning the selection criterion for support in Public-Private-Partnerships in LMICs [25-27]. Where supervision is provided it is perceived as more punitive than supportive. This makes the health workers suspicious and fearful and will make them evade the supervisory visits. Harassment and arrests of health workers by regulatory authorities has previously been reported by other researchers in Uganda [28].

While the support needed by the LLPHF is contextual and may vary from simple materials to use in the health facilities to funding for infrastructural improvement, both policy makers and health care providers agreed that all the facilities need capacity building by training and technical supportive supervision. Other studies carried out in Uganda and other LMICs have demonstrated improvement in quality of care when support is given to lower level facilities [28-32] while Hill et al demonstrated that a supervision strategy with a supportive approach was most effective in getting good results, even though these studies mainly considered community health workers [33]. Some LLPHF lack basic items for provision of first line lifesaving services and therefore, material support is also crucial. Lack of basic materials in small private health facilities has previously been described in studies in central Uganda [34]. 
Delegating technical support to the HSD is a possible solution to handle challenges of accessibility. Since members of HSD live in the same locality of the LLPHF, they are physically accessible and in addition, they are part of the community and thus suspicion and fear is overcome compared to supervisors coming from the $\mathrm{DHO}$ or $\mathrm{MOH}$ headquarters. Studies show that health workers at the peripheral facilities can be trained to effectively offer support supervision to lower level facilities $[28,29]$.

The strength of this study lies in the fact that we employed qualitative methods and explored the perspectives of both health workers and policy makers on support rendered to low level private clinics. We included a broad range of policy makers from highest to the lowest level of decision making and supervision as well as a variety of health workers. Failure to interview and capture views of the health regulatory councils and other regulatory bodies was a limitation. We therefore were not able to confirm some of the findings from LLPHF such as harassment from regulatory bodies.

\section{Conclusions}

Our study has shown that the current support being received by LLPHF is inadequate and identified bureaucracies, misinformation and inaccessibility of facilities and limited resources as barriers for providing the needed support. The support needs to be tailored to the needs of the facility and health facilities have to be proactive and ask for support however, capacity building with emphasis on training and supportive supervision are key strategies.

We recommend that clinic owners endeavour to bring their clinic to the attention of the policy makers through registration with the relevant professional councils and submission of regular performance reports as required by the $\mathrm{MoH}$; this will facilitate proper planning by policy makers to seek for and distribute available resources. Facilities should be organised and join the different organisations already recognised by the $\mathrm{MOH}$ such as religious federations and bureaus through which support can be channelled. The $\mathrm{MOH}$ should encourage Medical Bureaus to open up to the small private health facilities and show them how to access the support that already exists from implementing partners. To overcome the challenge of lack of human resources and inaccessibility of health facilities, the District Health Team should mentor and delegate some duties of support supervision to the HSDs. By regulating the number of facilities in the district to a manageable level, and supporting only facilities that register annually, the authorities can ensure sustainability of the support. This will hopefully weed out the illegal facilities that provide poor quality services as clients will chose facilities offering quality services. The MOH should take the lead in guiding partners to align their agendas with the needs of the community instead of accepting the partners to dictate.

\section{Abbreviations}

DHO

district health office; HC:health centres; HSD:health sub-districts; LLPHF:low level private health facilities; LMICs:low and middle income countries; $\mathrm{MOH}$ :Ministry of Health; NDA:National Drug Authority; 
NGOs:non-government organisations; SOMREC:School of Medicine Research and Ethics Committee; SDG:Sustainable Development Goal; RAs:research assistants; UNCST:Uganda National Council for Science and Technology

\section{Declarations}

\section{Ethics approval and consent to participate}

Ethical clearance to carry out the research was sought and received from the Makerere University, School of Medicine Research and Ethics Committee (SOMREC) referenced \#REC REF 2017-059 and the Uganda National Council for Science and Technology (UNCST) referenced SS 4903. To ensure anonymity providers at private clinics were de-identified by use of study numbers. For policy makers where gender and employment position lead to disclosure of participant's identity we have opted not to include the gender of the respondents in the quotes in order to further de-identify them. To ensure confidentiality of study data, transcripts and the voice recordings were transferred to a password protected locked computer only accessed by principal investigator and co-investigators. Printed documents such as signed consent forms were kept in a locked cabinet accessible only to the principal investigator.

\section{Consent for publication}

All participants consented to publication or other forms of dissemination of the results

\section{Availability of data and materials}

The data that support the findings of this study are available from Juliet Mwanga-Amumpaire but restrictions apply to the availability to maintain confidentiality of some of the respondents, and so are not publicly available. Data are however available from the authors upon reasonable request.

\section{Competing interests}

None of the authors has any competing interest to declare

\section{Funding}

This research was funded by Makerere-SIDA (MAK-SIDA) bilateral research program. The funders had no role in the study design, data collection and analysis, decision to publish nor in preparation of manuscript.

\section{Authors' contributions}

JMA conceived the idea, protocol development, data collection and quality assurance, coding and analysis of data, manuscript writing. JK: protocol development, manuscript writing. KK: protocol development, manuscript review. RS: data analysis and manuscript writing. JO: coding and data analysis, manuscript review. CO: protocol development, manuscript review. TA: protocol development, manuscript 
writing. GN: protocol development, manuscript writing. All authors have read and approved the manuscript.

\section{Acknowledgements}

We acknowledge MAK-SIDA for funding, Patricia Tushemereirwe, Beatrice Katusiime and Collins Ogola for data collection, Grace Nambozi for guidance with data coding. We also acknowledge all the health workers and policy makers who accepted to be interviewed and the owners of the private facilities who allowed the interviews to take place in their premises.

\section{Availability of data and materials}

The data and materials used in this manuscript can be availed by author Juliet Mwanga-Amumpaire on request.

\section{References}

1. UNICEF, et al., Levels and trends in child mortality 2015. 2015, UNICEF,WHO,World Bank,United Nations. p. 36.

2. You D., et al., Global, regional, and national levels and trends in under-5 mortality between 1990 and 2015, with scenario-based projections to 2030: a systematic analysis by the UN Inter-agency Group for Child Mortality Estimation. The Lancet, 2015. 386(10010): p. 2275-2286.

3. UNICEF, UNICEF data: Monitoring the situation of children and women; Trends in under-five mortality rate, UNICEF, Editor. 2018.

4. Uganda, M.O.H., Annual Health Sector Perfomance Report 2016/2017, M.o.H. Uganda, Editor. 2017.

5. Ministry of Heath, U., Annual Health Sector Perfomance Report. 2014, Ministry of Health, Uganda: Kampala.

6. Uganda, M.o.H., Annual Health Sector Performance Report Financial Year 2017/2028. 2017/2018, Ministry of Health.

7. UBOS, SELECTED INDICATORS, LIBERATION DAY CELEBRATIONS JANUARY 26, 2018 in UGANDA BUREAU OF STATISTICS PUBLICATION. 2018.

8. Ssennyonjo, A., et al., Government resource contributions to the private-not-for-profit sector in Uganda: evolution, adaptations and implications for universal health coverage. International journal for equity in health, 2018. 17(1): p. 1-12.

9. Mackintosh, M., et al., What is the private sector? Understanding private provision in the health systems of low-income and middle-income countries. The Lancet, 2016. 388(10044): p. 596-605.

10. MinistryofHealth, HealthSystems20/20, and MakerereUniversitySchoolofPublicHealth, Uganda Health System Assessment 2011. 2012, Ministry of Health, Uganda and Bethesda, MD: Health Systems 20/20 project, Abt Associates Inc. Abt Associates: Kampala, Uganda. 
11. Konde-Lule, J., et al., Private and public health care in rural areas of Uganda. BMC international health and human rights, 2010. 10(1): p. 29.

12. Rutebemberwa, E., et al., Utilization of public or private health care providers by febrile children after user fee removal in Uganda. Malaria Journal, 2009. 8(1): p. 45.

13. Basu, S., et al., Comparative Performance of Private and Public Healthcare Systems in Low-and Middle-Income Countries: A Systematic Review. PLOS Medicine, 2012. 9(6): p. e1001244.

14. English, M., et al., Explaining the effects of a multifaceted intervention to improve inpatient care in rural Kenyan hospitals-interpretation based on retrospective examination of data from participant observation, quantitative and qualitative studies. Implementation science : IS, 2011. 6(1): p. 124.

15. Gathara, D., et al., Quality of hospital care for sick newborns and severely malnourished children in Kenya: A two-year descriptive study in 8 hospitals. BMC Health Services Research, 2011. 11(1): p. 307.

16. Vukoja, M., et al., A Survey on Critical Care Resources and Practices in Low- and Middle-Income Countries. Global Heart, 2014. 9(3): p. 337-342.e5.

17. MOH, National Health Facility Master List 2018, A complete list of all health facilities in Uganda. 2018 ed. Vol. 2018. 2018, Kampala, Uganda: Ministry Of Health, Uganda. 164.

18. Morse, J.M., Determining sample size. 2000, Sage Publications Sage CA: Thousand Oaks, CA.

19. Creswell, J. and J.W. Creswell, Qualitative inquiry and research design.(2007). Qualitative inquiry \& research design: Choosing among five approaches.

20. Hsieh, H., dan S. Shannon, S. 2005. Three approaches to qualitative content analysis. Qualitative Health Research. 15(9): p. 1277-1288.

21. Strauss, A. and J. Corbin, 1990, Basics of Qualitative Research, Grounded Theory Procedures and Techniques, Newbury Park CA: Sage Publications. 1998.

22. Willcox, M.L., et al., Circumstances of child deaths in Mali and Uganda: a community-based confidential enquiry. The Lancet Global Health, 2018. 6(6): p. e691-e702.

23. (ISER), I.f.S.a.E.R., "Achieving Equity in Health: Are Public Private Partnerships the Solution? 2019, ISER: Kampala, Uganda.

24. Miyawaki, A. and Y. Kobayashi, Effect of a medical subsidy on health service utilization among schoolchildren: A community-based natural experiment in Japan. Health Policy, 2019. 123(4): p. 353359.

25. Ravishankar, N., et al., Private capacity, public payment: private business participation in government initiatives to improve access to critical health services. BEAM Exchange, 2016.

26. Suchman, L., E. Hart, and D. Montagu, Public-private partnerships in practice: collaborating to improve health finance policy in Ghana and Kenya. Health policy and planning, 2018. 33(7): p. 777785.

27. Yadav, V., et al., Facilitators and barriers to participation of private sector health facilities in government-led schemes for maternity services in India: a qualitative study. BMJ open, 2017. 7(6): p. 
e017092.

28. Kisakye, A.N., et al., Effect of support supervision on maternal and newborn health services and practices in Rural Eastern Uganda. Global health action, 2017. 10(sup4): p. 1345496.

29. Ajeani, J., et al., A cascade model of mentorship for frontline health workers in rural health facilities in Eastern Uganda: processes, achievements and lessons. Global health action, 2017. 10(sup4): p. 1345497.

30. Agarwal, R., et al., Improving quality of care during childbirth in primary health centres: a steppedwedge cluster-randomised trial in India. BMJ global health, 2018. 3(5): p. e000907.

31. Imani, P., et al., Effect of integrated infectious disease training and on-site support on the management of childhood illnesses in Uganda: a cluster randomized trial. BMC pediatrics, 2015. 15(1): p. 103.

32. Namazzi, G., et al., Strengthening health facilities for maternal and newborn care: experiences from rural eastern Uganda. Global health action, 2015. 8(1): p. 24271.

33. Hill, Z., et al., Supervising community health workers in low-income countries - a review of impact and implementation issues. Global Health Action, 2014. 7(1): p. 24085.

34. Buregyeya, E., et al., Comparison of the capacity between public and private health facilities to manage under-five children with febrile illnesses in Uganda. Malaria Journal, 2017. 16: p. 183.

\section{Figures}




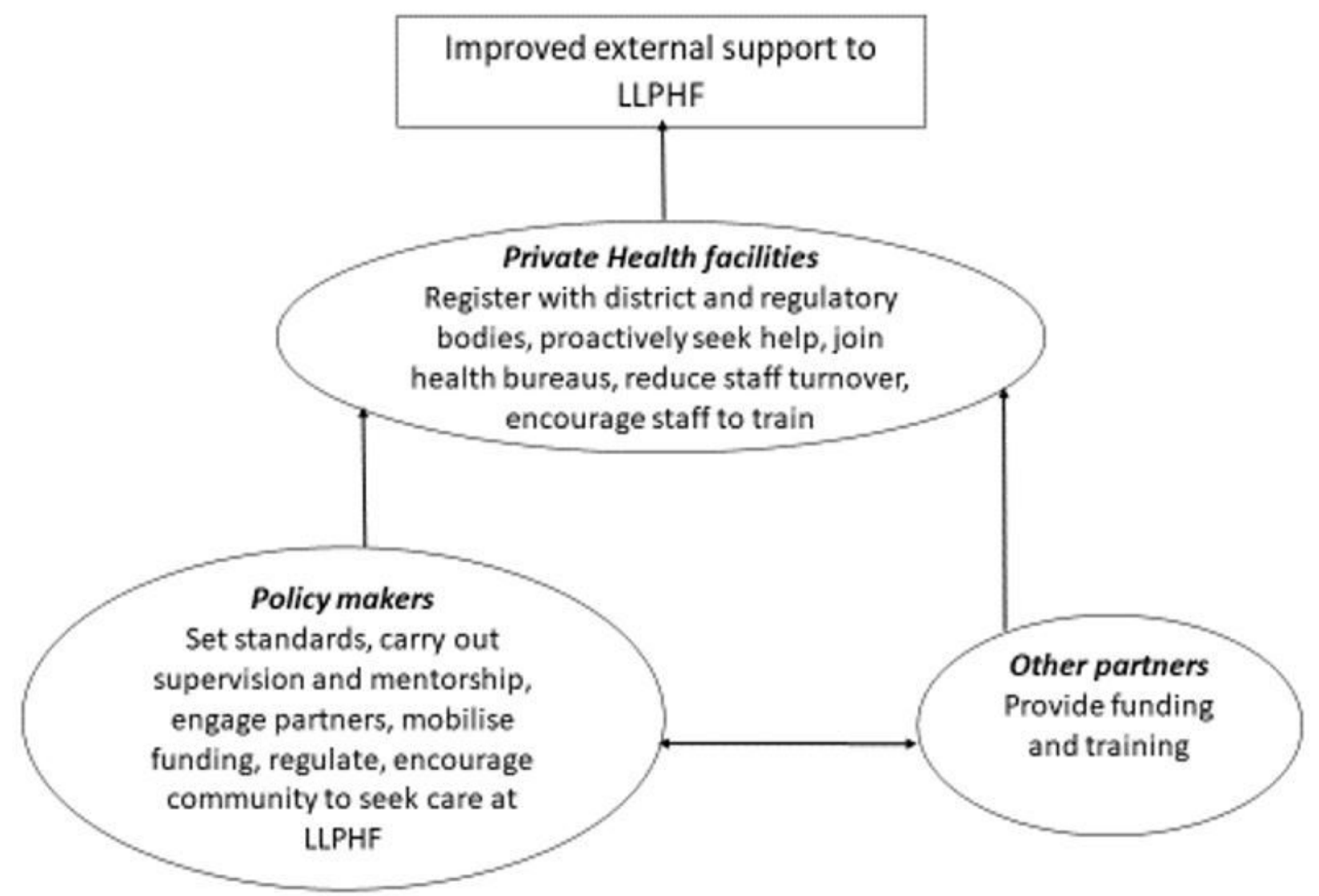

Figure 1

Organisation of the preferred modalities for improving external support to LLPHF

\section{Supplementary Files}

This is a list of supplementary files associated with this preprint. Click to download.

- COREQchecklist.pdf

- InterviewguideMOHleaders.pdf

- InterviewguideDisctricthealthleaders.pdf

- Interviewguidehealthworkers.pdf 